\title{
ESPACIOS EMOCIONALES Y MEDICINA EN EL SIGLO XIX. UNA ONTOLOGÍA HISTÓRICA DE LAS PASIONES
}

\author{
Juan Manuel Zaragoza Bernal \\ Universidad de Murcia \\ Email: Jm.zaragozabernal@um.es \\ ORCID iD: https://orcid.org/0000-0001-8377-6688
}

Recibido: 5 abril 2020; Aceptado: 25 septiembre 2020

Cómo citar este artículo/Citation: Zaragoza Bernal, Juan Manuel (2021) “Espacios emocionales y medicina en el siglo xix. una ontología histórica de las pasiones", Asclepio, 73(1): p341. https://doi.org/10.3989/asclepio.2021.07

RESUMEN: Las nuevas concepciones en la comprensión de las emociones desarrolladas en las dos primeras décadas del siglo XXI posibilitan el desarrollo de nuevos programas de investigación en humanidades y ciencias sociales que tengan en cuenta los elementos de la cultura material y el entorno social en la constitución de la experiencia emocional. Desde este punto de partida, en el presente artículo se realiza, empleando las herramientas de la ontología histórica, el análisis de uno de los hospitales para enfermos incurables, crónicos y ancianos situados en Madrid en el siglo XIX, caracterizándolo como un espacio emocional, es decir: aquellos en los que la gestión de las emociones es la principal práctica médica. Para ello analizaremos las propuestas teóricas desarrolladas por Durand-Fardel y Charcot acerca del tratamiento de este tipo de afecciones. Emplearemos los conceptos de marco y espacio enmarcado, de Erving Goffman, como herramienta explicativa que nos permita comprender la tarea de estructuración de la experiencia emocional puesta en práctica, lo que nos permitirá definir herramientas conceptuales que favorezcan la reinterpretación de las fuentes disponibles desde una nueva perspectiva.

PALABRAS CLAVE: lan Hacking; Enfermedad incurable; Experiencia emocional; Espacio emocional; Erving Goffman.

\section{EMOTIONAL SPACES AND 19TH CENTURY MEDICINE. A HISTORICAL ONTOLOGY OF PASSIONS.}

ABSTRACT: A new understanding of emotions has been developed in the first two decades of the 21st century that make it possible to develop new research programs in the humanities and social sciences that take into account the role played by material culture and social environment in the constitution of emotional experiences. From this starting point, this article analyses a $19^{\text {th }}$ century hospital for incurable, chronic and elderly patients based in Madrid, characterizing it as an emotional space, that is: one in which managing emotions was the main medical practice. For this, we will analyse the theoretical proposals developed by Durand-Fardel and Charcot regarding these conditions. We will use the concepts of frame and framed space, by Erving Goffman, as an explanatory tool that will allow us to understand medical practice structured the emotional experience of the inmates. We will define conceptual tools that will help us to analyse available sources from a new perspective, that of historical ontology.

KEY WORDS: Ian Hacking; Incurable Disease; Emotional Experience; Emotional Space; Erving Goffman. 


\section{INTRODUCCIÓN}

El estudio de las emociones en la historia se ha convertido en una de las más importantes corrientes historiográficas de los últimos 20 años, que ha alcanzado ya su madurez (Zaragoza 2013; Boddice 2017; Bjerg 2018). A lo largo de este proceso, sin embargo, han ido apareciendo problemas e inconsistencias que, al acumularse, empiezan a poner en cuestión algunas de las asunciones básicas en que esta se asentaba (Moscoso 2015; Boddice 2019 y 2020). Entre las críticas destaca aquella que cuestiona una suerte de "sentido común" acerca de las emociones que estaría basado en una cierta concepción de estas como "clases naturales", que sería compartido de forma generalizada por la mayoría de investigadores sobre emociones, y que funcionaría como un paradigma transdisciplinar (Barrett, 2006; Boddice, 2018). Los resultados obtenidos en el estudio del cerebro en los últimos años, sin embargo, socaban esta concepción, hasta el punto de que algunos proponen un cambio de paradigma hacia otro en el que la función de la cultura en la conformación de nuestras emociones sería fundamental. Es lo que se ha llamado la teoría de las emociones construidas (Barrett, 2017). Esta nueva aproximación, que escapa a la manida distinción naturaleza/cultura, ofrece a las humanidades, y en particular a la historia, una excelente oportunidad para replantearse su aportación al estudio de las emociones y explorar nuevas propuestas metodológicas.

Con este artículo buscamos contribuir a dicho esfuerzo. Nos basamos, para ello, en la propuesta de lan Hacking de la ontología histórica (Hacking, 2002) y, en concreto, en su concepción de las clases interactivas o humanas (Hacking, 1995). Como ya hemos señalado otras veces (Zaragoza, in press), si las emociones ya no son clases naturales, como sostiene Barret, podemos considerarlas clases humanas o interactivas, es decir: aquellas que aplicamos sobre seres humanos y que, al hacerlo, cambian la forma en que nos pensamos a nosotros mismos, mediante lo que Hacking denominó "efecto bucle". Tal y como defendemos, esta forma de entender las emociones es congruente con el nuevo paradigma propuesto por Barrett y nos permite desarrollar un proyecto de investigación innovador en el campo de la historia de las emociones. Este artículo pretende contribuir a dicho desarrollo, a través del estudio de las condiciones materiales que participan en la gestión y construcción de las emociones de grupos sociales localizados (confinados) en un espacio compartido.
Nos centramos para ello en un estudio de caso localizado en el Hospital para Hombres Incurables Nuestra Señora del Carmen de Madrid en 1891, tal y como se recoge en la documentación que se guarda en el Archivo Histórico Nacional. Se trata de una colección fragmentaria e incompleta, que se encuentra dentro del Fondo Contemporáneo, dentro de los documentos correspondientes al Ministerio de Gobernación, que ostentaba en ese periodo las competencias de Beneficencia. Para profundizar en la comprensión de las implicaciones de nuestro caso de estudio, recurriremos a la psicología de la época, ejemplificada de forma sobresaliente en el influyente Curso de Psicología y Lógica de Pedro Felipe Monlau y Jose María Rey y Heredia, así como a otras obras del periodo especializadas en el tratamiento y las condiciones de los internos en dichas instituciones, tanto nacionales como extranjeras. Además, se hará uso de diversas obras de higiene social para dar cuenta de la relevancia de las condiciones materiales, tal y como nos muestra Joaquim Salarich, en su Higiene del tejedor o nuevamente Monlau en sus diversas obras sobre higiene. También atenderemos a la obra de reformadores sociales, como Concepción Arenal, que dedicó a estos hospitales de incurables una serie de artículos y reflexiones, así como a lo que podríamos denominar "la psicología del pobre".

\section{CÓDIGOS DISCIPLINARIOS Y GESTIÓN EMOCIONAL}

En enero de 1891, en el Hospital para Hombres Incurables Nuestra Señora del Carmen de Madrid, la Junta de Caridad, compuesta por señoras de la alta sociedad de la época como la marquesa de Martorell o la condesa de Toreno, se reúne para imponer una sanción a los internos Hipólito Moreno y Eduardo Plana. La sanción, en este caso, será ejemplar, y llega incluso a ir en contra de los reglamentos aprobados apenas cinco años antes y publicados en la Gazeta de España. Se castiga a estos internos a cuatro meses de reclusión en el recinto del hospital y a la pérdida del privilegio de recibir visitas. Pero no queda ahí el veredicto de la Junta de Damas, sino que va más allá cuando recomienda que, en caso de que los internos reincidan en la misma falta, sean expulsados inmediatamente del hospital. Sorprende el castigo por su dureza y, como decíamos, por contradecir incluso el reglamento de la institución, que en su capítulo XXIV tan solo permitía la "supresión de paseos y recreos", siempre y cuando no perjudicaran a la salud del enfermo, como forma de castigo (junto a la amonestación en privado y en público y la expulsión del centro). El castigo, por tanto, rozaba los límites reglamentarios y, en cualquier caso, sí que aconsejaba aplicar la medida de mayor dureza contra Hipólito Moreno y Eduardo Plana: la expulsión ${ }^{1}$. 
No podemos dejar de pensar que la dureza del castigo debe corresponderse a la gravedad de la falta, pero, ¿qué falta habían cometido Hipólito y Eduardo? La respuesta es, cuanto menos, desconcertante. La privación de visitas y paseos durante cuatro meses, así como la recomendación de expulsión del centro, responden al hecho de que Hipólito Moreno y Eduardo Plana fueron sorprendidos jugando a las cartas en el centro. Que esta fuera la falta que merecía un castigo tan duro no puede menos que llamarnos la atención. Se antoja desproporcionado, incluso cruel, comparado con la gravedad de aquella. Y, sin embargo, el Comité tiene razones para imponer tal castigo. Razones que expone: el juego, nos dicen, provoca violentas pasiones en los internos. La sentencia de la Comisión, por tanto, nos sitúa en el terreno de la gestión emocional. Lo que se castiga no es el juego en sí, que obviamente no es "una buena costumbre", sino el motivo por el que se juega: la alteración de las emociones que se produce en los jugadores. El hospital se convierte en gestor de las emociones de sus internos ${ }^{2}$. Al prohibir el juego lo que se pretende es evitar las pasiones que el juego levanta, y que pueden causar daño a los enfermos y a la institución. Esta gestión de las emociones se consigue convirtiendo al hospital en un espacio emocional, concepto que se definirá en el tercer apartado del presente texto (Gammerl, 2012; Pernau, 2014). Para llegar a tal definición será necesario ver qué pasiones son las que levanta el juego y por qué son tan peligrosas para los internos del Hospital Nuestra Señora del Carmen.

\section{JUEGO Y DESPOSESIÓN DEL YO}

En Los jugadores (Ilustración 1), Simón Gómez Polo (1845-1880) -pintor de la escuela catalana que realizó esta obra en el año 1870, actualmente en el Museo de Arte Moderno de Barcelona-, presenta, sobre un fondo oscuro, a tres ancianos jugando a los dados mientras otro, más joven, los observa. Sobre la mesa, una frasca de vino, los dados, la mano del que lanza y unas monedas. Curiosamente, no hay vasos. El lanzador, con la cabeza ladeada, mira fijamente al personaje situado a la izquierda, que lleva sus manos a la cabeza. Es una mirada de superioridad, del que sabe que ha derrotado completamente al otro. El tercero en discordia, al fondo, fuma su pipa mientras contempla al que ha realizado la jugada. El observador, de pie, reclinado hacia la mesa, con pinta de lechuguino, atiende el juego con una sonrisa en la boca y mirada divertida... A la izquier$\mathrm{da}$, aislado de los tres que conforman un grupo, encontramos al otro jugador, un tercer anciano que presenta un aspecto totalmente desesperado, sosteniendo la cabeza con la mano izquierda. Suponemos que el dinero que hay sobre la mesa es suyo y, también, que la jugada que acaba de salir a su contrincante significa que lo ha perdido. El pañuelo en la mano derecha, que descansa en su rodilla, nos permite imaginar que ha secado el sudor de su frente más de una vez, señal de un nerviosismo recurrente. Todo el cuadro está marcado por las emociones de los representados: la profunda desesperación del perdedor, la condescendencia y el orgullo del ganador, la indiferencia del fumador, la curiosidad del joven. Si el Comité disciplinario del Nuestra Señora del Carmen señalaba las "violentas pasiones" que el juego suscita, el cuadro de Gómez Polo se detiene en algunas de ellas. Pasiones violentas que, en muchas ocasiones, pueden terminar en violentos altercados (Campos Marín, 1997).

Ilustración 1: Simón Gómez Polo (1845-1880). Els jugadors de daus (1874). Óleo sobre tela. 74,5 x 100,5 $\mathrm{cm}$. Museo Nacional d’Art de Catalunya. Licencia: dominio público.

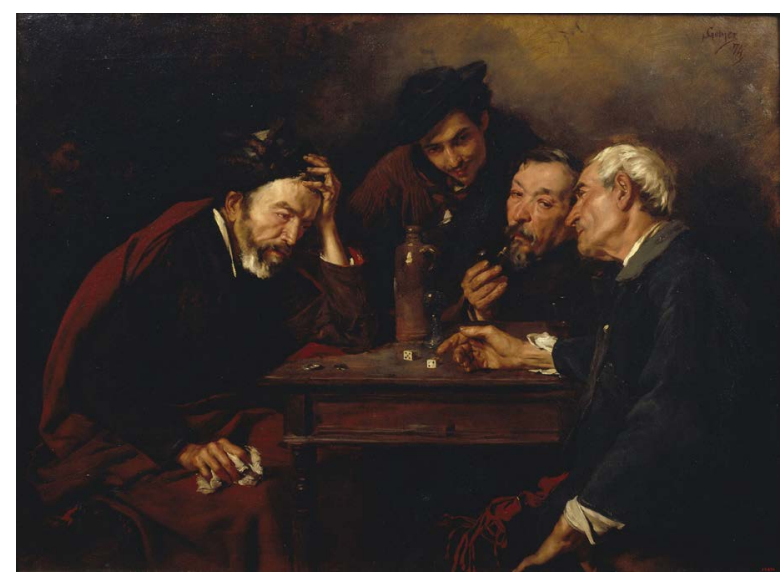

El juego será por tanto peligroso para los internos por dos motivos principales. En primer lugar, porque el juego, por sí mismo, enciende las pasiones hasta el punto de que el jugador puede llegar a perder todo control sobre sí mismo. La pasión por jugar empuja al implicado a apostar todo lo que lleva encima. El juego se convierte aquí en el puente a través del cual las emociones de los implicados irrumpen en la escena, emociones que hacen perder la razón a los implicados, hasta que estos pierden todo control sobre las propias acciones, que están dirigidas por las pasiones desatadas por la apuesta. Esta pérdida de voluntad tan solo puede conducir al jugador a un final trágico.

En su curso de psicología, Monlau y Rey distinguen tres facultades del alma, a las que debe el hombre ser persona y no cosa, esas facultades o potencias son: la sensibilidad, la inteligencia y la voluntad (Monlau y Rey y Heredia 1866, 1:21). De estas tres facultades, las dos primeras se distinguen por ser pasivas, es decir, 
no tenemos conciencia directa de ellas, sino que suponemos su existencia por sus efectos. La voluntad, sin embargo, es activa, en el sentido de que somos conscientes de ella (Monlau y Rey y Heredia 1866, 1:22). La voluntad es, para Monlau, la base del Yo, de la subjetividad:

De la oposición que acabamos de hacer notar, resulta que la voluntad es, como dice Descartes, lo más propiamente nuestro que hay en nosotros; ó, más bien, la voluntad es nosotros mismos, y ella sola constituye, por decirlo así, la persona humana (Monlau y Rey y Heredia, 1866, 1:23).

La pérdida de la voluntad implica, por tanto, una pérdida del yo, puesto que "la voluntad es el YO" (Monlau y Rey y Heredia, 1866, 1:24), "la voluntad constituye la vida personal" (Monlau y Rey y Heredia, $1866,1: 81)$. La voluntad implica una posesión de sí mismo, contrapunto pasivo de la actividad volitiva, e imprescindible para que esta se produzca (sin ser parte de ella, que se define por la resolución) (Monlau y Rey y Heredia, 1866, 1:80). Esta posesión de uno mismo explicaría las diferencias de los caracteres. En efecto, si la voluntad es igual en todos los seres humanos, tal y como indica Monlau, la diferencia entre caracteres firmes y débiles estribará en la extensión e intensidad de uso de la voluntad; algo que depende de esa posesión de sí mismo como contraparte pasiva de la volición. Lo que hemos llamado "pérdida de voluntad" en el juego sería en realidad, según Monlau, una desposesión de uno mismo. No se trata, de una cuestión de clases sociales, sino de un problema estrechamente relacionado con la constitución de la subjetividad ${ }^{3}$. No obstante, resulta pertinente señalar que, pese a que en principio cualquiera puede ser víctima de la pasión por el juego, serían las clases "pobres" las que mayor riesgo correrían, puesto que en ellas la fuerza de la voluntad es más débil:

[...] el miserable que se asusta al hablarle del trabajo, y que prefiere la miseria a la ocupación útil, pasa días y noches enteras en el fondo de una taberna entreteniendo su espíritu con las emociones del juego de azar, perdiéndose con sus compañeros de orgía en este abismo sin fondo [...] (Salarich 1984, 205) ${ }^{4}$

Esta última cita de Salarich introduce el segundo elemento que convierte al juego en un peligro: el lugar donde este tiene lugar, un ambiente de depravación y delito, tabernas donde el alcohol y las prostitutas son parte permanente del paisaje, y el baratero impone su ley. En El Capitán de la Chesnaye, folletín de Ernesto Capendu publicado en Barcelona en 1861, y publicado por entregas, posteriormente, por La Correspondencia de España entre 1879 y 1880, encontramos un claro ejemplo de estos ambientes patibularios en los que el juego se desarrolla. En la entrega del 19 de enero de 1880 , Capendu nos relata el ambiente de salón de juego donde entran los personajes:

Aquellos dos que juegan a los dados diciéndose mil injuriosas frases, amenazándose con el puño y con la daga, gritando y dando alaridos a cada jugada, son Juan Horca y Santiago el Chocarrero, los acólitos de maese Jonás [...] Por todas partes, en fin, comen, beben, gritan, peroran, aúllan, ladran, maúllan, lloran, ríen, disputan, se abrazan, se pelean, blasfeman, se cocean en medio de un concierto horrible formado por clamores espantosos [...] (Capendu, 1880).

Hay mucho de descripción estereotipada y de género (literario) en este pasaje, no obstante, es precisamente esta asociación entre juego y una suerte de "incontinencia emocional" lo que subyace a la sentencia del Comité, una asociación construida sobre materiales no mucho menos estereotipados que el presentado en este folletín. En esta misma dirección apunta Ricardo Campos en su análisis del discurso burgués sobre la taberna:

[...] se observa una excesiva uniformidad y abstracción en su tratamiento, propiciada por la incapacidad burguesa de eludir los tópicos y los prejuicios, en la descripción de los mismos. La imagen de la taberna no parece superar una serie de rasgos que la definen como antihigiénica e inmoral (Campos Marín 1997, 179).

La taberna, lugar en el que la pasión por el juego es liberada, es el lugar en el que las clases más desfavorecidas tienen acceso a las costumbres más depravadas y donde dan rienda suelta a sus más bajos instintos. La literatura médica del XIX señala precisamente a la taberna, en tanto que opuesta a la familia y al hogar, como el lugar en que se originaban todos los males de las clases más bajas (Campos Marín 1997, 184):

El pobre empieza a ir a la taberna en busca de sociedad y de distracción; hay gente, conversación animada, se juega, etc, etc. Una vez allí, bebe, convida, le convidan, se anima, bebe más, para demostrar que tiene dinero que aguanta mucho, por emulación, que la tiene el vicio como la virtud. Se le pasa el tiempo agradablemente, se aficiona a ir, cada día bebe más, y le repugnan menos las cosas repugnantes que allí ve y oye, de modo que, al cabo de algún tiempo, es un vicioso o un criminal, o entrambas cosas, según varias circunstancias unas personales suyas, y otras de los que le rodean (Arenal, 1911)..$^{5}$ 
La taberna se asocia, por tanto, con la delincuencia, el alcoholismo, la prostitución y la revuelta obrera (Campos Marín 1997, 165), es decir: con todos los vicios posibles en una clase, la de los pobres, que estaba predispuesta a ellos por una falla en la constitución de la subjetividad de sus miembros. El higienismo toma en consideración otros muchos elementos, como son la organización del trabajo, la miseria, las condiciones de vida de los llegados a la ciudad para trabajar en la industria, la herencia, etc. (Campos Marín, Pérez, y Huertas 2000, 166). Sin embargo, estas condiciones no son sino elementos que se oponen a la voluntad. El mérito consiste, precisamente, en la realización del esfuerzo necesario para vencer estos obstáculos (Monlau y Rey y Heredia, 1866, 1:74). De ahí que hubiera "pobres buenos", como los hubiera calificado Concepción Arenal, y también era esta la razón que justificaba el mito de la movilidad en la escala social, tan bien descrito por Galdós en sus novelas de Torquemada (Pérez Galdós 2008), pero también el hecho de que el caer en el vicio fuera, en última instancia, una responsabilidad individual. $Y$ de entre todos los vicios reunidos en el espacio de la taberna, el juego es uno de los que mayor impacto tiene sobre ella:

Allí, alrededor de una mala mesa, junto a una copa de vino comprado con los ahorros de su familia, pasa el vagabundo jugador la parte más florida de sus años [...]no goza del sol, de la luz, ni de los placeres sociales, porque su incuria y pasión le condenan a vivir aislado de todo afecto; porque sus amigos, y su familia cuya desventura causa, llegan a aborrecerle y abandonarle a sus pasiones favoritas, a los compañeros de juego [...] entre quienes no tarda en embrutecer su alma, pervertir su moral y convertirse en objeto repugnante, de menosprecio y peligroso para la sociedad (Salarich, 1984, 205-6).

El juego entre las clases populares se relaciona, como muestra este texto y otros similares, con un vicio y una degeneración que tiene como mejor representación la expresión violenta y descontrolada de las pasiones ${ }^{6}$. Esta "incontinencia emocional" del jugador bien puede ser una marca de clase, pero el juego hace aflorar fácilmente las pasiones como un distintivo propio de su misma naturaleza: si las clases populares no tienen gran control sobre sus pasiones, el juego es el terreno idóneo que permite que se desborden ${ }^{7}$. Por otra parte, el juego en las clases populares se encuentra estrechamente relacionado con un espacio de sociabilidad que escapa a los controles de la burguesía (Campos Marín 1997, 157), que se contrapone a la vida familiar (y moral), y que es el origen de gran parte de los problemas que representa este sector de la población. La taberna, espacio demonizado por la medicina, se opone frontalmente al espacio que nuestros dos internos, Hipólito Moreno y Eduardo Plana, habitan. Un espacio que, por tanto, no puede admitir el juego entre sus muros.

\section{MARCOS EMOCIONALES}

Volvamos al cuadro de Gómez Polo. Quisiéramos señalar un segundo elemento: la composición misma del cuadro, el instante del juego. Como vemos, los cuatro integrantes de la escena se encuentran situados en medio de la oscuridad, aislados del resto del entorno, como si a su alrededor se hubiera creado un marco de sombras, entre las que entrevemos, pero sin que llegue a formar parte de la escena, el rostro desconocido de quien debe ser, con bastante probabilidad, la tabernera. Lo realmente significativo de este cuadro, lo que consigue atrapar la habilidad del pintor, no es, en realidad, la escena costumbrista de tres ancianos jugando a los dados en una taberna del último cuarto del XIX, sino la esencia misma del juego: el hecho de que jugar sitúa a los jugadores en un marco experiencial distinto al que habitaban antes. Sus actos (mover la mano, situar unas monedas sobre la mesa, sostener un pañuelo) se interpretan de forma distinta dentro de este nuevo marco que nos permite interpretar lo que está ocurriendo. Esta franja de experiencia (Goffman 2006, $11)^{8}$ congelada en el cuadro de Gómez Polo solo puede ser interpretada si tenemos en cuenta el hecho de que "están jugando". El juego, por tanto, implica un cambio de marco. El marco de experiencia anterior al juego ya no está vigente, ahora solo es posible interpretar las acciones de los protagonistas dentro del nuevo marco. Y más aún, los jugadores solo pueden vivir la experiencia que el nuevo marco les "permite" vivir. Goffman define el marco, en su obra Frame Analysis, como "aquel que se considera que convierte en algo que tiene sentido lo que de otra manera sería un aspecto sin sentido de la escena" (Goffman, 2006, 23). En esta dirección, ante la escena pintada por Gómez Polo, lo que confiere sentido a lo que está ocurriendo es que identificamos el marco en que los personajes se insertan. Al identificar que están jugando, aspectos como las monedas sobre la mesa o el movimiento de la mano del lanzador adquieren un significado que, si no fuera por ese marco, escaparía de nosotros. Los marcos no solo proporcionan sentido al observador, sino que también lo hacen a los que se encuentran en su interior: el marco permite dar sentido a la experiencia vivida al insertarla dentro de unas coordenadas culturales, al proporcionar un sistema de reglas. El participante en el marco, por tanto, moldeará su experiencia para responder a las coordenadas proporcionadas por el marco en que se inserta, actividad que, al mismo 
tiempo, construye el marco. Sin esta interacción entre marco y participante sería imposible dotar de sentido a la acción ${ }^{9}$.

El juego, por tanto, se define como un marco que confiere sentido a determinadas franjas de experiencia. Como hemos visto, parte del contenido del marco "juego" consiste en lo que hemos llamado "incontinencia emocional". Entre los conjuntos de reglas (más o menos claras) que componen los marcos, se incluyen normas sobre la gestión emocional. Así, la asistencia a un funeral implica una serie de normas sobre las emociones que el asistente debe sentir y expresar, unos límites para esa expresión de las emociones, etc. EI juego, como se ha visto en los ejemplos anteriores, se caracteriza por dejar un amplio margen para la expresión de las emociones. No nos extraña que un jugador grite de alegría cuando realiza una excelente jugada al lanzar los dados, mientras que, fuera del marco del juego, encontraríamos extraño el mismo entusiasmo por el hecho de arrojar dos cubos sobre una mesa con un movimiento de la mano izquierda.

La idea de "marco emocional", que haría referencia a aquellos elementos que regulan la presencia de emociones dentro de una actividad dada, nos permitirá entender la importancia que para los miembros del comité disciplinario del Nuestra Señora del Carmen tiene prohibir el juego dentro de las salas del hospital. En los siguientes apartados introduciremos dos nuevos conceptos que ayudan en la interpretación del suceso histórico: el espacio enmarcado (framed space) y la atmósfera.

\section{ESPACIOS EMOCIONALES: EL SILENCIO DE LAS SIMPATÍAS}

Así pues, el motivo para prohibir el juego no es el juego en sí, sino esa "incontinencia emocional" que hemos descrito. ¿Por qué es tan importante, en un centro como el Nuestra Señora del Carmen, evitar situaciones de incontinencia emocional? Para dar respuesta a esta pregunta debemos acudir a las obras médicas sobre las enfermedades crónicas y de la vejez del siglo XIX. De entre ellas, cabe destacar dos obras de origen francés y traducidas al castellano entre 1875 y 1885 . La primera de ellas es el Tratado práctico de las enfermedades crónicas, de Maxime Durand-Fardel (Durand-Fardel, 1877), publicado originalmente en 1854 . Esta es, sin lugar a dudas, la primera gran obra sobre las enfermedades crónicas y de la vejez que se publica en Europa en el siglo XIX. El segundo libro está escrito en 1866 y está firmado por Jean-Marie Charcot, con el título Lecciones clínicas sobre las Enfermedades de los Viejos y las Enfermedades Crónicas, y se publica en España en el año 1883, como el anterior en la Biblioteca escogida de Cirugía y Medicina, bajo la dirección de Méndez Álvaro (Charcot, 1883).

Lo primero que cabe destacar es la proximidad de las categorías que vamos a manejar. Las enfermedades de la vejez, las crónicas y las incurables son, durante el siglo XIX, prácticamente una misma cosa. Las razones con que se explican, las prácticas médicas que las rodean, los discursos que intentan delimitarlas son, casi punto por punto, idénticos. En este caso, son dos los elementos que nos interesan. El primero es la incapacidad de la medicina para curar estas enfermedades. La misma definición de enfermedad incurable (aquellas que no se pueden curar) (Zaragoza, 2012), lo indica. Las enfermedades de la vejez dependen del estado orgánico del cuerpo anciano $\mathrm{y}$, como tales, no pueden ser curadas a no ser que se revierta el proceso de envejecimiento, algo a todas luces imposible. Por tanto, si no pueden ser curadas, nos dice Charcot, lo único que está en manos del médico es paliarlas. El libro de Charcot, que presta gran atención a la gota y al reumatismo articular, señala la incapacidad de los medios utilizados en la medicina para la curación de estos males crónicos (Charcot, 1874, 234), así como su relativa incapacidad para paliar sus síntomas. En estos casos, nos dice Charcot, debemos dar la primacía a "los modificadores higiénicos" (Charcot, 1874, 234). De la misma opinión es Durand-Fardel (Durand-Fardel, 1854, XLIII). Ante la incapacidad de la medicina para curar y paliar los efectos de las enfermedades crónicas y propias de la vejez, y en tanto que estas son constitutivas, la práctica médica que debe primar en estos casos es la higiene.

El segundo punto que es necesario resaltar acerca de este tipo de enfermos tiene que ver, precisamente, con su "inconsistencia emocional". La vejez, el proceso de envejecimiento, consiste en una serie de cambios que se producen en el organismo. Estos cambios, ambos médicos coinciden, pueden entenderse como un "aislamiento de los órganos entre sí" (Durand-Fardel, 1854, XXXIX; Racle, 1864, 647-748; Charcot, 1874, 10), que es el origen de las características más relevantes de las patologías de la vejez: la escasez de síntomas, la falta de fiebre, la muerte súbita sin causa aparente. Este proceso de separación tiene, como una de sus muchas consecuencias, un proceso paralelo que separa al anciano del mundo: el debilitamiento de los sentidos hace que la percepción se oscurezca, que la realidad pierda sus contornos al tiempo que los sentidos se retiran y el sistema nervioso se debilita (Durand-Fardel, 1854, XXXII). El anciano se separa del mundo de la misma forma que sus órganos se separan entre sí, 
en una perfecta simetría entre lo interior y lo exterior. A este proceso lo llama Durand-Fardel "el silencio de las simpatías" (Durand-Fardel, 1854, XL). El prolífico y conocido médico e higienista francés, Victor Alexandre Racle, señala claramente en qué consiste ese silencio:

[...] la indiferencia por los objetos exteriores y por la humanidad se establece y crea un estrecho egoísmo que convierte a la vejez en algo odioso. De ahí que no acaezcan en la vejez aquellas enfermedades mentales que derivan de la exaltación de las facultades afectivas: esas facultades ya no existen (Racle, 1864, 647).

Esta separación del mundo convierte al anciano en un ser débil, que puede morir por exponerse a una ráfaga de aire frío en una iglesia (Durand-Fardel 1854, XLIV). De ahí que el tratamiento higiénico idóneo consista en controlar las condiciones en que se produce el contacto con el exterior, de forma que sea dentro de unos límites que el anciano -el enfermo- pueda tolerar (Durand-Fardel, 1854, X). Estas mismas restricciones rigen en el plano emocional. El enfermo crónico incurable, el anciano, es presa de las más terribles emociones, que pueden minar su alma "volviéndolo sombrío y egoísta" (Descuret 1844, 34; Monlau, 1864, 491; González Fernández, 1906, 54) ${ }^{10}$. "Las enfermedades crónicas hacen el carácter inquieto, sombrío, egoísta e irascible", dice Descuret en su Patología de las pasiones (Descuret, 1844, 70). Este estado emocional debe ser, por tanto, objeto de tratamiento, tanto espiritual (González Fernández, 1906, 185), como médico (Grenet, 1903, 315). El tratamiento de la moral del enfermo -del anciano-, la restricción de la influencia de las pasiones en el curso de su enfermedad y en la evolución de su alma son parte fundamental, por tanto, de su cuidado. Y para ello, nos dice Grenet, nada mejor que "mantenerlo ocupado, hacerle seguir un pequeño reglamento" (Grenet 1903, 215). No en vano, como bien dice Monlau: "Los efectos de las pasiones son terribles. Como en su esencia no son más que transgresiones higiénicas, pueden producir todas las enfermedades conocidas" (Monlau, 1864, 381). Y eso, precisamente, es lo que el Nuestra Señora del Carmen pone a disposición de sus internos: un espacio en el que se toman una serie de medidas (disciplinarias, higiénicas, morales, etc.) que buscan -entre otros muchos objetivos-, mantener un determinado registro emocional.

\section{ESPACIO ENMARCADO}

A lo largo de su carrera, Goffman prestó una especial atención a los elementos físicos que formaban parte de la experiencia y que ayudaban a comprenderla ${ }^{11}$. En La presentación de la persona en la vida cotidiana,
Goffman señala la importancia que, para la presentación del individuo, tiene su atrezzo, o, como él lo designa, su apariencia (Goffman, 1971, 35). Pero el elemento que capta nuestra atención en la metáfora teatral tiene que ver con el papel de la escena, el medio en que el individuo representa su papel, y que influye en el éxito de la misma:

En primer lugar, se encuentra el medio (setting), que incluye el mobiliario, el decorado, los equipos y otros elementos propios del trasfondo escénico, que proporcionan el escenario y utilería para el flujo de acción humana que se desarrolla ante, dentro o sobre él (Goffman, 1971, 34).

El medio, nos dice Goffman, forma parte de la representación, y se sitúa en regiones, esto es, "todo lugar limitado, hasta cierto punto, por barreras antepuestas a la percepción" (Goffman, 1971, 117). Se establece así una primera relación entre el espacio y el marco. En este caso, el espacio forma parte del marco, proporcionando posibilidades de interpretación. Pero existe una segunda opción, que merece la pena ser explorada: aquella en que marco y espacio coinciden.

Goffman distingue entre regiones delanteras y traseras, de acuerdo con el acceso que el "público" tiene a ellas. Si bien puede haber excepciones, se da el caso de que tan solo la región anterior es accesible, mientras que la posterior (backstage) queda oculta a la mirada de los espectadores. En cada una de estas regiones, las posibilidades de actuación del personaje varían, así como las normas que rigen dicha actuación (Goffman, 1971, 118-23). Las distintas regiones son utilizadas para que el espectador interprete la "realidad" de la forma en que el intérprete ha escogido, haciendo coincidir el espacio y el marco de interpretación. El siguiente ejemplo de Goffman ilustra magníficamente esta coincidencia:

Si el personal de un hospital para enfermos mentales debe lograr que quienes visitan a sus allegados reciban una opinión favorable del establecimiento, será entonces importante impedir a los visitantes el acceso a las salas, en especial a las destinadas a enfermos crónicos, limitando su estadía a salas especiales de visitas, donde será factible disponer de un mobiliario agradable y asegurarse de que todos los pacientes presentes se hallen bien vestidos, limpios, bien cuidados y se comporten con relativa corrección (Goffman, 1971, 125).

Ahora bien, esto nos permite asociar un único marco a un determinado espacio escénico. Será posible, por supuesto, encontrar diversos marcos funcionando dentro de la misma región, pero dicha región estará 
diseñada para contener o acentuar un único marco escogido por el equipo de actuantes. Esto debe incluirse en la lista de limitaciones de la metáfora dramatúrgi$\mathrm{ca}^{12}$. Resulta evidente para cualquiera que un espacio puede ser diseñado para contener más de un marco. El caso más evidente tal vez sea una iglesia, donde debemos interpretar el acto de compartir el pan y el vino de una forma especial y, al tiempo, la configuración espacial tiende a organizar una determinada jerarquía social, dependiendo de la proximidad del público al altar. Encontramos dentro del mismo espacio, por tanto, como mínimo dos marcos interpretativos de lo que allí está ocurriendo (Goffman, 2006, 137-38). Marcos que se apoyan en la constitución física del espacio, si bien solo uno -el primero en este caso- es explícitamente sancionado durante la construcción del mismo.

Una de sus últimas aportaciones a la relación entre interpretación de la experiencia y espacio fue el concepto de framed space, que introduce en su último libro Forms of talk (Goffman, 1981, 230). Goffman define este concepto al hablar de los diversos formatos de la programación radiofónica y cómo estos imponían un marco interpretativo a las palabras del hablante que, junto a otro tipo de restricciones dependientes del papel que interpretase en ese momento (locutor principal, invitado, etc.), podían llamarse su "espacio marco", dentro del cual sus palabras tendrían sentido. Como siempre ocurre con Goffman, este marco no es absoluto. Es posible obviarlo, pero esto tendrá consecuencias en el proceso de comunicación. El espacio enmarcado conforma, así, la red de elecciones y expectativas del hablante ( $y$ del oyente, que también posee su propio campo), conformando sus posibilidades de experiencia. El espacio enmarcado lo restringe a la hora de expresar una cosa u otra, y también le advierte de las consecuencias que tendría obviar esas restricciones. En un estudio del año 1976, Fredric Jameson calificaba los framed spaces goffmanianos como un simple recurso retórico (Jameson, 2000, 51), sin embargo, en conjunción con los argumentos desarrollados en La presentación del yo en la vida cotidiana, nos permite hablar de espacios que se constituyen para contener diversos marcos de interpretación y excluir otros. Espacios que se organizan para contener una determinada estructuración de la experiencia. Podemos entonces identificar espacios restrictivos (cuando tan solo se permiten unos determinados marcos, como es el caso de la institución total), o abiertos, cuando permiten la presencia de varios marcos al mismo tiempo, algunos de los cuales pueden ser incluso contradictorios (como puede ser el caso de una plaza pública) ${ }^{13}$. Espacios, en definitiva, creados para contener diversos marcos que funcionan al mismo tiempo, de forma más o menos coordinada. Este sería el caso de un hospital, en el que diversos marcos se superponen (terapéutico, administrativo, legal, etc.). Puede darse el caso de que uno de esos marcos sea considerado el principal, siendo el resto secundarios y, en teoría, al servicio de aquel. La relación entre estos marcos no sería inalterable, pudiendo darse el caso de que en determinadas circunstancias alguno de los marcos secundarios pasara a ser principal.

Entre los marcos que forman parte, o pueden formar parte, de este espacio enmarcado, se encuentra el que hemos llamado, al principio del presente apartado, marco emocional. Todo espacio enmarcado contiene un determinado marco emocional, que puede ser más o menos relevante. Así, por retomar el ejemplo de la iglesia, se supone que todo aquel que entra en un templo deberá sentir una serie de emociones (alegría, arrepentimiento, constricción) y no otras (ira, avaricia, deseo), y se articulan toda una serie de prácticas para primar las primeras y "purgar" las segundas. El aspecto emocional será más o menos relevante, de la misma forma que lo eran el terapéutico y el administrativo, y su relación con el resto de marcos será más o menos conflictiva. También se dará el caso, cuando el marco emocional sea el principal en un espacio enmarcado dado, de que podamos calificar a ese espacio como un "espacio emocional". Así sucedería con el Hospital Nuestra Señora del Carmen.

Como hemos visto en las páginas anteriores, el único elemento capaz de tener un impacto positivo en los pacientes del hospital, una vez descartado el terapéutico y paliativo, tiene que ver con la gestión de las emociones de los internos. Para esa gestión, se desarrollan toda una serie de normas, disposiciones y prácticas encaminadas a situar a los ingresados en un determinado marco emocional, caracterizado por la ausencia de fuertes pasiones y el fomento de un estado de ánimo que podríamos calificar como contemplativo. En tanto que es esta la principal actividad que se desarrolla en el hospital, es decir, en tanto que ese marco emocional es el más relevante dentro de ese espacio enmarcado, podemos calificar al Hospital para Hombres Incurables Nuestra Señora del Carmen como un espacio emocional.

\section{CONCLUSIONES}

En el presente artículo defendemos una aproximación a la historia de las emociones basada en el análisis de las condiciones sociales y materiales en que estas se producen. Esta aproximación, que ya ha sido explorada con anterioridad (Gerhard Jaritz 2003; Parrott 
2005; Harris y Sørensen 2010; Labanyi 2010; Gammerl 2012; Pernau 2014; Styles 2015; Zaragoza 2015; Holloway 2019), adquiere toda su potencialidad cuando se adopta la teoría, propuesta por Barrett, de las emociones construidas, que surgirían de "una combinación entre las propiedades físicas del cuerpo, un cerebro flexible cuyas conexiones reflejan el entorno en el que se desarrolla, y la cultura y la educación que ofrecen ese entorno" (Barrett, 2018, 18). Un entorno que no podemos entender sin tomar en cuenta la cultura material.

Al aplicar esta nueva aproximación a la historia de nuestros dos internos en el Hospital Nuestra Señora del Carmen para Hombres Incurables de Madrid, hemos sido capaces de entender mejor cuáles eran las lógicas de funcionamiento detrás de la práctica médica de la reclusión aplicada a estos internos. En tanto que sus enfermedades no tenían cura, hemos sostenido que la principal función de estos hospitales no era otra que la de generar en sus internos una determinada experiencia emocional y hemos señalado que tal estructuración de la experiencia se conseguía a través de la implementación de marcos que dotaban de significado a las acciones realizadas dentro de ese espacio. Quedaría por desarrollar los elementos concretos que conforman el marco, lo que nos abriría nuevas posibilidades de interpretación de las fuentes materiales y

\section{NOTAS AL FINAL}

1 Archivo Histórico Nacional, Fondo Contemporáneo, Gobernación, Serie Beneficencia, Legajo: 2022/37.

2 La terminología empleada en la historia de las emociones sigue siendo, a día de hoy, uno de los temas candentes de la disciplina. El uso de pasiones, emociones, afectos... puede situarnos dentro de una corriente o tradición nacional determinada, con sus correspondientes vínculos teóricos e institucionales. En este artículo hemos decidido utilizar estos términos como si fueran sinónimos, empleándolos de forma intercambiable, para que la discusión terminológica no nos aparte de nuestro objetivo principal: entender el papel que la cultura material tiene en la configuración de nuestra experiencia emocional.

3 “ '...] la pasión del juego. Si comúnmente toma origen en la ociosidad de la opulencia, nace también a veces de la miseria y de los disgustos, del trato con los caballeros de industria, del mal ejemplo, de la ocasión" (Monlau 1847, 2:784). Por su parte, Letourneau nos recuerda que Descartes "tuvo, durante su juventud, la pasión del juego sobre la que no triunfó más que a fuerza de voluntad y de filosofía" (Letourneau 1868, 56). El más certero acercamiento a la psicología española del siglo XX y a sus concepciones acerca de la subjetividad en (Novella, 2013). visuales, un aspecto que exploraremos en un artículo posterior.

Para concluir, queremos volver a señalar la importancia del cambio de paradigma sobre el que nuestra propuesta se asienta. La teoría de las emociones construidas inserta a la cultura como un elemento fundamental en la constitución misma de nuestras emociones. No se trata de que module nuestra percepción emocional, ni de que dé forma a lo que conocemos como "emociones complejas", sino que son parte fundamental en el proceso de composición emocional. Esto ofrece, tanto a humanistas como a científicos sociales, la posibilidad de contribuir de forma fundamental, y en pie de igualdad, al estudio transdiciplinar de las emociones.

\section{AGRADECIMIENTOS}

Trabajo realizado en el marco de los Proyectos de Investigación "Improvisación y contagio emocional. Historia y teoría de experiencias emocionales" (PID2019-108988GB-I00) del Ministerio de Ciencia e Innovación y "La naturaleza como horizonte ético. Modernidad, crisis ecológica y experiencia del orden", financiado por el Programa Renovación Generacional para el Fomento de la Investigación (RG2020-004UM) de la Universidad de Murcia.

4 También Monlau insiste en la facilidad de los más pobres para caer en las garras del vicio: "La embriaguez, la gula, el juego, el libertinaje y la pereza, son las pasiones dominantes en los hombres; la prostitución y la estafa son los vicios y delitos que la policía y los tribunales se ven precisados á reprimir y castigar con más frecuencia en las obreras" (Monlau 1856, 85).

5 Citado en (Campos Marín 1997, 168-69).

6 Monlau nos dice que una de las causas principales por las que nos aproximamos al juego es "el anhelo de emociones variadas" (Monlau 1864, 403). Y también Salarich: "Las ardientes emociones del juego forman una de sus más gratas delicias" (Salarich 1984, 207).

7 El tema de la incontinencia emocional, que recibe aquí un tratamiento de clase, merecería también de un análisis de género, tal y como se nos ha hecho notar por uno de los revisores del artículo. En efecto, una lectura en clave de género resultaría especialmente interesante, y no descartamos llevarlo a cabo en futuras investigaciones. Sin embargo, creemos que escapa completamente de los límites del presente artículo.

8 Goffman utiliza el concepto "franja de experiencia" para referirse a "una fracción de actividad y secuencia de acontecimientos" (Chihu Amparán, 103).

9 "Permítaseme repetir que puesto que el marco incorpora tanto la respuesta del participante como el mundo al que está respondiendo, necesariamente interviene un elemen- 
to reflexivo en la visión perspicaz de los acontecimientos de cualquier participante" (Goffman 2006, 91).

10 Esta imagen de los ancianos "sombríos y egoístas" por causas fisiológicas tiene una larga historia. La tradición hipocrático-galénica sostiene que los ancianos "son proclives a la tristeza debido a la frialdad de su sangre" (Wright 1971, 73), citado en (Alberti 2010, 20).

\section{BIBLIOGRAFÍA}

Alberti, Fay Bound (2010), Matters of the Hearth. History, Medicine, and Emotion, Oxford, Oxford University Press.

Alberti, Fay Bound (2010), Matters of the Hearth. History, Medicine, and Emotion, Oxford, Oxford University Press.

Arenal, Concepción (1911), “La Taberna”, España Social 7: p. 3.

Barrett, Lisa Feldman (2006), "Are Emotions Natural Kinds?", Perspectives on Psychological Science 1 (1): 28-58, [en línea], https://doi.org/10.1111/j.1745-6916.2006.00003.x.

Barrett, Lisa Feldman (2017), "The Theory of Constructed Emotion: An Active Inference Account of Interoception and Categorization", Social Cognitive and Affective Neuroscience 12(1), pp. 1-23, [en línea], https://doi.org/10.1093/scan/ nsw154.

Barrett, Lisa Feldman (2018), La vida secreta del cerebro: Cómo se construyen las emociones, Barcelona, Ediciones Paidós.

Bjerg, Maria (2018), "Una genealogía de la historia de las emociones", Quinto Sol 23 (1), [en línea], https://doi. org/10.19137/qs.v23i1.2372.

Boddice, Rob (2017), The History of Emotions: Past, Present, Future", Revista de Estudios Sociales, 62, pp. 10-15, [en línea], https://doi.org/10.7440/res62.2017.02.

Boddice, Rob (2018), The History of Emotions, Manchester, Manchester University Press.

Campos Marín, Ricardo (1997), Alcoholismo, medicina y sociedad en España (1876-1923), Madrid, CSIC.

Campos Marín, Ricardo, José Martínez Pérez, y Rafael Huertas (2000), Los ilegales de la naturaleza. Medicina y degeneracionismo en la España de la Restauración (1876-1923), Madrid, CSIC.

Capendu, Ernesto (1880), “El Capitán de la Chesnaye”, La Correspondencia de España, 19 de enero de 1880

Charcot, Jean-Martin (1874), Leçons Cliniques sur les Maladies des Vieillards et les Maladies Chroniques, 2.a ed, Paris, Adrien Delahaye.

Charcot, Jean-Martin (1883), Lecciones Clínicas sobre las Enfermedades de los Viejos y las Enfermedades Crónicas, Madrid, Enrique Teodoro.

Descuret, J.B.F. (1844), La médecine des passions ou Les passions considérées dans leurs rapports avec les maladies, les lois et la religión, Paris, Labé.
11 Tal vez su categoría más lograda sea la de institución total, que desarrolla en (Goffman 1961).

12 Sobre las limitaciones de la metáfora dramática, ver (Goffman 1971, 270)

13 Sobre la constitución del espacio como acto político, el referente es (Lefebvre 2013)

Durand-Fardel, Maxime (1854), Traité Clinique et Pratique des Maladies des Vieillards, Paris, Germer Baillière.

Durand-Fardel, Maxime (1877), Tratado Práctico de las Enfermedades Crónicas., Biblioteca Escogida de «El Siglo Médico», Madrid, Enrique Teodoro.

Gammerl, Benno (2012), “Emotional Styles - Concepts and Challenges”, Rethinking History 16 (2), pp. 161-175.

Goffman, Erving (1961), Asylums: Essays on the Social Situation of Mental Patients and Other Inmates, Chicago, Aldine Pub. Co.

Goffman, Erving (1971), La presentación de la persona en la vida cotidiana, Buenos Aires, Amorrortu Editores.

Goffman, Erving (1981), Forms of Talk, Oxford, Basil Blackwell.

Goffman, Erving (2006), Frame Analysis. Los marcos de la experiencia, Madrid, Siglo XXI / CSIC.

González Fernández, Marcelino (1906), Visita de enfermos Asistencia de moribundos ó sea Manual teórico-práctico del sacerdote para el ejercicio de aquel ministerio, Oviedo, Establecimiento Tipográfico «La Cruz».

Grenet, L. (1903), Arte de cuidar a los enfermos. Manual teórico práctico para uso de las familias en general y de las enfermeras religiosas en particular, Barcelona, Gustavo Gili.

Hacking, Ian (1995), "The Looping Effect of Human Kinds". En Sperber, Dan; Premack, David; Premack, Ann James (eds.), Causal Cognition: A Multidisciplinary Debate, New York: Oxford University Press, pp. 351-383.

Hacking, Ian (2002), Historical Ontology, Cambridge, Mass. \& London, Harvard University Press.

Harris, Oliver J. T., y Tim Flohr Sørensen (2010), "Rethinking Emotion and Material Culture", Archaeological Dialogues 17 , pp. $145-63$

Holloway, Sally (2019), The Game of Love in Georgian England: Courtship, Emotions, and Material Culture, Oxford, United Kingdom ; New York, Oxford University Press.

Jameson, Fredric (2000), “On Goffman's Frame Analysis". En Fine, Gary Alan; Smith, Gregory W.H. (eds.), Erving Goffman, London, SAGE Publications, pp. 43-55.

Jaritz, Gerhard (2003), Emotions and Material Culture : International Round Table-Discussion, Krems an Der Donau, October 7 and 8, 2002, Wien, Verlag der Österreichischen Akademie der Wissenschaften.

Labanyi, Jo (2010) "Doing Things: Emotions, Affect, and Materiality", Journal of Spanish Cultural Studies, 11, pp. 223-33. 
Lefebvre, Henri (2013), La producción del espacio, Madrid, Capitán Swing.

Letourneau, Charles Jean Marie (1868), Physiologie des passions, Paris, G. Baillière.

Monlau, Pedro Felipe (1847), Elementos de Higiene Pública, Barcelona, Imprenta de D. Pablo Riera.

Monlau, Pedro Felipe (1856), Higiene Industrial. ¿Qué medidas higiénicas puede dictar el gobierno a favor de las clases obreras? Madrid, Imprenta y Estereotipia de M. Rivadeneyra.

Monlau, Pedro Felipe (1864), Elementos de higiene privada o arte de conservar la salud del individuo, Madrid, Librería de Moya y Plaza.

Monlau, Pedro Felipe, y Jose María Rey y Heredia (1866), Curso de Psicología y Lógica, Madrid, M. Rivadeneyra.

Moscoso, Javier (2015), "La historia de las emociones, ¿de qué es historia?", Vínculos de Historia. Revista del Departamento de Historia de la Universidad de Castilla-La Mancha, 4, [en línea], http://vinculosdehistoria.com/index.php/vinculos/ article/view/147.

Novella, Enric (2013), La ciencia del alma. Locura y modernidad en la cultura española del siglo XIX, Madrid, Frankfurt am Main, Vervuert Verlagsges.

Parrott, Fiona (2005), “"It's Not Forever»: The Material Culture of Hope", Journal of Material Culture, 10, pp. 245-62.

Pérez Galdós, Benito (2008), Las novelas de Torquemada, Las Palmas de Gran Canaria, Cabildo de Gran Canaria.

Pernau, Margrit (2014), "Space and Emotion: Building to Feel", History Compass 12 (7), 541-549.
Racle, V.A. (1864) Traité de Diagnostic Médical ou Guide Clinique por l'étude des Signes Caractéristiques des Maladies, Paris, J.B. Bailliere et Fils.

Salarich, Joaquim (1984) «Higiene del Tejedor. O sean, medios físicos y morales para evitar las enfermedades y procurar el bienestar de los obreros ocupados en hilar y tejer el algodón». En Jutglar, Antonio (ed.), Condiciones de vida y trabajo obrero en España a mediados del siglo XIX, Barcelona, Anthropos.

Styles, John (2015), “Objects of Emotion: The London Foundling Hospital Tokens, 1741-60", En Gerritsen, Anne y Riello, Giorgio (eds.), Writing Material Culture History, London, Bloomsbury Academic, pp. 165-72.

Wright, Thomas (1971), Passions of the Minde in Generall, Urbana, II, University of Illinois Press.

Zaragoza, Juan Manuel (2012), “La palabra más brutal: definiciones de la enfermedad incurable en la medicina francesa del siglo XIX", Asclepio 64 (2), pp. 491-516.

Zaragoza, Juan Manuel (2013), "Historia de las emociones: una corriente historiográfica en expansión”, Asclepio, 65 (1), [en línea] https://doi.org/10.3989/asclepio.2013.12.

Zaragoza, Juan Manuel (2015), “Ampliar el marco. Hacia una historia material de las emociones", Vínculos de Historia. Revista del Departamento de Historia de la Universidad de Castilla-La Mancha, 4, [en línea], http://vinculosdehistoria. com/index.php/vinculos/article/view/148

Zaragoza, Juan Manuel (in press), "Investigación filosófica y emociones: la ontología histórica de lan Hacking como alternativa", Daimon. 